\title{
Hemodynamic Comparison Between Right Heart Catheterization and Transthoracic Echocardiography in the Critically Ill Patients: A Prospective Study
}

\author{
Hassan Khouli ${ }^{*}, 1$, Edward Eden ${ }^{1}$, Peter Homel ${ }^{3}$ and Mark V. Sherrid ${ }^{2}$
}

\author{
Divisions of ${ }^{1}$ Pulmonary and Critical Care and ${ }^{2}$ Cardiology, St. Luke's-Roosevelt Hospital Center, Columbia University \\ College of Physicians and Surgeons, New York, NY, ${ }^{3}$ Maimonides Medical Center, New York, NY, USA
}

\begin{abstract}
Background: Tranthoracic echocardiography (TTE) is increasingly utilized in the management of critically ill patients in whom right heart catheterization (RHC) was frequently used in the past. We tested the hypothesis that bedside TTE can substitute for RHC for determining the etiology of cardiopulmonary compromise in critically ill patients when initial clinical assessment is limited.

Methods: We prospectively enrolled 51 patients from medical and surgical Intensive Care Units with unknown etiology of cardiopulmonary compromise. Patients underwent assessment of their cardiopulmonary compromise by RHC and TTE. A final clinical assessment, considered the gold standard was adjudicated by the intensivist caring for the patient.

Results: There was complete agreement between TTE data and RHC data in determining the etiology of cardiopulmonary compromise as cardiac or non-cardiac in $46(90 \%)$ of the 51 patients. The kappa statistic for the agreement between TTE data and gold standard in determining the etiology of cardiopulmonary compromise as cardiac or non-cardiac was 0.90 ( $95 \%$ confidence interval [CI], 0.73 to $0.98 ; \mathrm{p}<0.001)$. The kappa statistic for the agreement between RHC data and gold standard was $0.84(95 \%$ confidence interval [CI], 0.63 to $0.95 ; \mathrm{p}<0.001)$. Based on the results of the gold standard assessment, the positive predictive value and negative predictive value for TTE data determining the etiology of cardiopulmonary compromise were $93 \%$ and $97 \%$ respectively. The positive predictive value and negative predictive value for RHC data determining the etiology of cardiopulmonary compromise were $100 \%$ and $92 \%$ respectively. TTE was highly suggestive of pulmonary embolism and cardiac tamponade in three patients where RHC was not.

Conclusion: In this era where transthoracic echocardiography is increasingly utilized to manage critically ill patients with cardiopulmonary compromise, we found transthoracic echocardiography to be a useful diagnostic tool in determining the etiology of cardiopulmonary compromise when initial clinical assessment is limited. While both methods can be complementary to each other, bedside transthoracic echocardiography is an acceptable non-invasive alternative to right heart catheterization in determining the etiology of cardiopulmonary compromise in most critically ill patients when initial clinical assessment is limited.
\end{abstract}

Keywords: Echocardiography, right heart catheterization, cardiopulmonary compromise.

\section{INTRODUCTION}

The management of hemodynamically unstable patients requires accurate assessment of the cardiovascular system. Since clinical assessment (CA), based on history, physical examination, laboratory, and radiographic evaluation, is limited [1], a pulmonary artery catheter (PAC) has traditionally been used to guide management. Measurement of left ventricular (LV) filling pressures and cardiac output may indicate whether the current cardiopulmonary compromise results from a cardiac or non-cardiac cause.

Despite more than 30 years of experience with the PAC, little evidence exists to demonstrate an outcome benefit in critically ill or peri-operative patients [2-5]. Routine use of the PAC is no longer indicated [6] because of the errors

*Address correspondence to this author at the Section of Critical Care, St. Luke's-Roosevelt Hospital Center, Columbia University College of Physicians and Surgeons, 1000 10th Avenue, New York, NY 10019, USA; Tel: 212-523-8914; Fax: 212-523-8822; E-mail: hkhouli@chpnet.org inherent in extrapolation of pressure to volume, the lack of uniform and accurate interpretation of data acquired by the PAC, and concerns over catheter-related complications. An alternative approach is the use of bedside TTE to provide "snapshot" assessment of cardiac function and determine if the etiology of cardiopulmonary compromise is related to cardiac or non-cardiac cause [7, 8]. In this era where RHC is less frequently used and intensivists are increasingly utilizing TTE to manage critically ill patients with cardiopulmonary compromise, we designed a study to validate whether current TTE equipment is adequate and can substitute for RHC for determining the etiology of cardiopulmonary compromise in critically ill as cardiac or non-cardiac and can provide initial management strategy when the initial clinical assessment is limited.

\section{MATERIALS AND METHODOLOGY}

\section{Patient Population}

All patients admitted to MICU and SICU with cardiopulmonary illness were screened for enrollment. 
Patients who met study inclusion criteria were consecutively enrolled in the study. Inclusion Criteria: 1) age > 17 years; and 2) acute cardiovascular compromise defined as shock (mean arterial pressure $<65 \mathrm{~mm} \mathrm{Hg}$ ) requiring vasopressors with evidence of tissue hypoperfusion (signs of lactic acidosis, impaired mental status, or oliguria defined as 0.5 $\mathrm{mL} / \mathrm{kg}$ /hour for $>2$ hours), or acute pulmonary compromise defined as new diffuse bilateral infiltrates on CXR with a $\mathrm{PaO} 2 / \mathrm{FIO} 2$ ratio $<300$ with or without mechanical ventilation support; and 3) lack of response to a trial of empiric therapy (fluid boluses up to 2 liter of normal saline and or short trial up to two hours of vasopressors or diuretics; and 4) unclear etiology of cardiopulmonary compromise concluded by the intensivist caring for the patient.

\section{Study Design}

We conducted a prospective study at a universityaffiliated, 850-bed, urban teaching hospital during a period of 3-years. The study was approved by the hospital Institutional Review Board. Patient informed consent was obtained. Fig. (1) illustrates study flow and essential measurements.

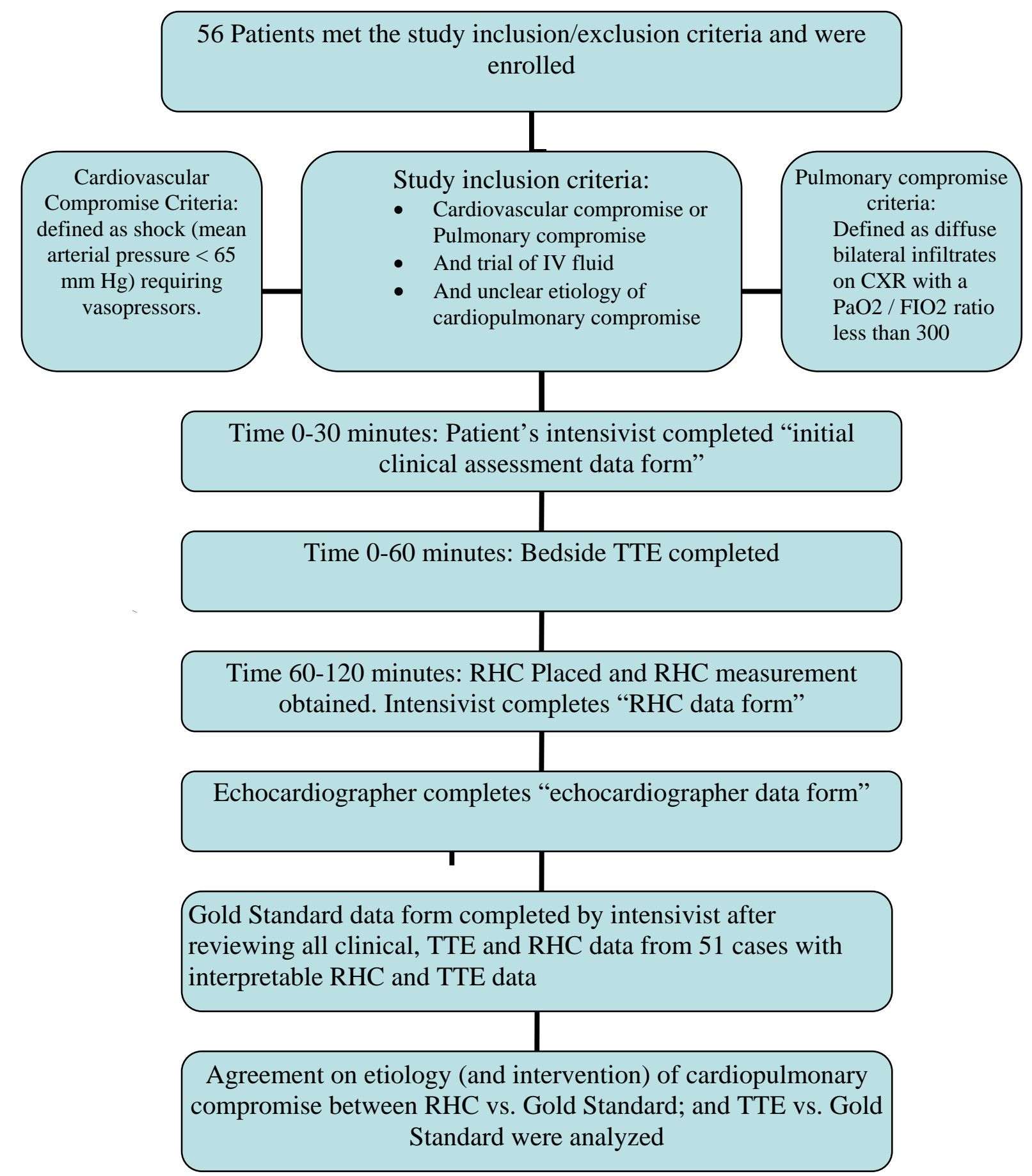

Fig. (1). Study flow and measurement variables. 
An echocardiographer blinded to the patient clinical assessment and RHC parameters was informed if the patient had cardiovascular, pulmonary compromise or both. He then reviewed the TTE study and completed an "echocardiographer assessment data form". An intensivist completed "RHC data form" after reviewing RHC parameters. Intensivists were critical care board certified and have reviewed The Pulmonary Artery Catheter Education Project (PACEP) tutorials. Final etiology of cardiopulmonary compromise was made by the patients' intensivist after reviewing clinical data, RHC parameters, and TTE assessment. This was considered the "gold standard" (GS) assessment. In cases of discrepancy between RHC and TTE measurements, one senior intensivist not involved in the care of the patients reviewed all data and adjudicated the final GS assessment. Gold standard diagnosis of cardiac etiology of cardiopulmonary compromise was established by the following results: 1) agreement between RHC and TTE data on cardiac etiology and review of both data elements and quality, 2) review of clinical history, ECG, and hospital course supporting cardiac etiology of cardiopulmonary compromise, 3) response to initial treatment initiated by the patient's intensivist based on data obtained from RHC and TTE, 4) autopsy and surgery results when available. A gold standard diagnosis of non-cardiac etiology of cardiopulmonary compromise was concluded if: 1) above observations were not consistent with cardiac etiology, 2) review of laboratory and microbiology culture results including serum lactate level were not consistent with cardiac etiology of cardiopulmonary compromise and an alternate diagnosis was confirmed. Measures of flow adequacy such as $\mathrm{SvO} 2, \mathrm{ScvO} 2$, and serum lactate level were reviewed by the intensivist and included in the assessment of the etiology of cardiopulmonary compromise and recommended initial treatment strategy.

Data collected in all three data forms included an assessment of the etiology of cardiopulmonary compromise as cardiac or non-cardiac, final diagnosis, and recommended initial management strategies based on preexisting guidelines that included the following options for treatment: additional fluids bolus, add or increase vassopressors, add inotropic agent, give diuretics, or none, according to the data obtained from RHC or TTE.

\section{Bedside TTE Study Protocol}

Echocardiograms in the intensive care unit were performed on Acuson Sequoia 256 Cardiac Ultrasound by a board certified echocardiography technician. Patients were positioned, with nursing assistance, in the left lateral decubitus position when tolerated and standard two dimensional, pulsed and color Doppler images from the parasternal long and short axis views, the apical 4, 3 and 2 chamber views and the subxiphoid views were recorded on videotape. 2.0 and $2.5 \mathrm{MHz}$ transducer frequencies were used for two dimensional imaging.

Two dimensional echocardiogram and color Doppler: Sonographer gave particular attention was made to LV size and function, RV size and function, and valvular regurgitation. Measurement of left ventricular end diastolic volume (LVEDV) was recorded. Global left ventricular ejection fraction (LVEF) was estimated visually by a previously validated method [9]. Severity of mitral regurgitation was estimated by the area of the left atrium occupied by the regurgitant jet and by corroborating data, such as proximal flow acceleration or mean proximal isovelocity surface area, reversal of pulmonary venous velocities, width of the vena contracta, and antegrade mitral inflow velocities.

Pulsed and Continuous wave Doppler: Pulmonary artery pressure was estimated as $4 \mathrm{~V}^{2}+$ the right atrial pressure, where $\mathrm{V}=$ the peak trans-tricuspid systolic velocity. Right atrial pressure (RAP) was estimated for quantification of pulmonary artery pressure. The inferior vena cava (IVC) was measured within $2 \mathrm{~cm}$ of the IVC-RA junction and IVC collapsibility with respiration was assessed. In spontaneously breathing patients IVC diameter was taken as the maximum measurement, and in ventilated patients it was taken in expiration. Patients with IVC diameter $>2 \mathrm{~cm}$ were assigned a RAP of 15-20 mm Hg and those with IVC diameter $<2 \mathrm{~cm}$ were assigned a RAP of $5-10 \mathrm{~mm} \mathrm{Hg}$. Variation of the diameter of the IVC with respiration was utilized as an adjunct to this estimation [10-13]. Whenever technically feasible, pulsed Doppler of pulmonary vein flow was recorded, from the apical four chamber view [14]. Mitral E and $\mathrm{A}$ wave velocities were assessed in each patient, as well as $\mathrm{E}$ :A ratio and transmitral $\mathrm{E}$ wave deceleration time. This data was used to supplement the pulmonary vein tracings that were the primary modality used to estimate LV filling pressure. In ventilated patients, these measurements were made during expiration, without breath hold. Isovolumic relaxation time and PV-mitral A duration were not utilized in this study because of a perception that limited information would be derived from the first parameter, and because of technical difficulties obtaining this measurement in the latter parameter in the critically ill.

\section{Echocardiography Assessment Criteria}

Cardiac etiology of cardiopulmonary compromise was diagnosed when 3 of the following 5 criteria were present (see Table 1):

1-Decreased LVEF $<50 \%$, 2- Qualitative LV dilatation confirmed by quantitative LVEDV. 3- Decreased pulmonary venous systolic flow velocity compared with diastolic velocity indicating high left atrial pressure, 4- High filling pressures were also diagnosed from the transmitral diastolic flow when there was a restrictive filling pattern, with high transmitral E wave, E:A ratio $>2.5: 1$, and decreased $\mathrm{E}$ wave deceleration time of $<150 \mathrm{msec}$. Low or normal filling pressures were diagnosed when the pulmonary venous flow velocity ratio was normal (systolic $>$ diastolic velocity) and $\mathrm{E}: \mathrm{A}$ ratio was normal. In these latter patients lower $\mathrm{E}$ velocities suggested lower filling pressures [14, 15]. IVC diameter and changes during respiration were included in the assessment. 5- Severe mitral regurgitation. In patients with severe bilateral pulmonary infiltrates and marked LV hypertrophy, cardiac etiology - diastolic dysfunction - was diagnosed if there was also marked reversal of pulmonary vein velocities and restrictive transmitral flow velocities. Patients without these findings were characterized as noncardiac. Right ventricular pressure overload indicating increased pulmonary vascular resistance, and suggestive of large pulmonary embolism was diagnosed when there was 
Table 1. Right Heart Catheterization and Transthoracic Echocardiography Criteria for Diagnosing Cardiac Etiology of Cardiopulmonary Compromise

\begin{tabular}{|c|c|}
\hline Right Heart Catheterization Criteria & Transthoracic Echocardiography Criteria*: 3 of 5 Needed \\
\hline 1. Cardiac index $<2.41 / \mathrm{min} / \mathrm{m}^{2}$ & 1. Reduced Left Ventricular ejection fraction $<50 \%$ \\
\hline 2. Stroke Volume Index $<40 \mathrm{~mL} /$ beat $/ \mathrm{m}^{2}$ & $\begin{array}{l}\text { 2. Qualitative Left Ventricular dilatation confirmed by quantitative Left Ventricular } \\
\text { End Diastolic Volume. }\end{array}$ \\
\hline 3. Pulmonary Capillary Wedge Pressure $>18 \mathrm{mmHg}$ & $\begin{array}{l}\text { 3. Decreased pulmonary venous systolic flow velocity compared with diastolic } \\
\text { velocity indicating high left atrial pressure. }\end{array}$ \\
\hline 4. Central Venous Pressure $>12 \mathrm{~mm} \mathrm{Hg}$ & $\begin{array}{l}\text { 4. High filling pressures were also diagnosed from the transmitral diastolic flow } \\
\text { when there was a restrictive filling pattern, with high transmitral E wave, E:A } \\
\text { ratio }>2.5: 1 \text {, and decreased E wave deceleration time of }<150 \text { msec. Inferior } \\
\text { Vena Cava diameter and changes during respiration were included in the } \\
\text { assessment. }\end{array}$ \\
\hline 5. Systemic Vascular Resistance Index $>2400$ dynes.sec. $\mathrm{cm}^{5} / \mathrm{m}^{2}$ & 5. Severe mitral regurgitation. \\
\hline
\end{tabular}

*In patients with severe bilateral pulmonary infiltrates and marked LV hypertrophy, cardiac etiology - diastolic dysfunction - was diagnosed if there was also marked reversal of pulmonary vein velocities and restrictive transmitral flow velocities. Right ventricular pressure overload indicating increased pulmonary vascular resistance, and suggestive of large pulmonary embolism was diagnosed when there was systolic pulmonary hypertension $>45 \mathrm{~mm} \mathrm{Hg}$ associated with right ventricular dilatation, often with hypokinesia, and with compression of a normally functioning left ventricle. Pericardial tamponade was diagnosed in the presence of $>$ small pericardial effusion with diastolic compression of the right atrium and right ventricle with characteristic phasic respiratory variation of the mitral, tricuspid and aortic Doppler tracings.

systolic pulmonary hypertension $>45 \mathrm{~mm} \mathrm{Hg}$ associated with right ventricular dilatation, often with hypokinesia, and with compression of a normally functioning left ventricle $[16,17]$. Pericardial tamponade was diagnosed in the presence of $>$ small pericardial effusion with diastolic compression of the right atrium and right ventricle with characteristic phasic respiratory variation of the mitral, tricuspid and aortic Doppler tracings. Because of technical factors all the echocardiographic criteria could not be acquired in every patient. If incomplete data was present (not uncommon in clinical situations as well), the best diagnostic assessment possible was performed.

\section{Right Heart Catheterization Criteria}

All RHC pressure measurements were obtained at the end of expiration. Normal pulmonary capillary wedge pressure (PCWP) was a range of 10-18 mm Hg. Normal cardiac index was a range of $2.4-4.01 / \mathrm{min} / \mathrm{m}^{2}$. Normal LV stroke volume index (SVI) was a range of $40-70 \mathrm{~mL} / \mathrm{beat} /$ $\mathrm{m}^{2}$. Normal systemic vascular resistance index (SVRI) was a range of 1600-2400 dynes.sec. $\mathrm{cm}^{5} / \mathrm{m}^{2}$. Cardiac etiology of cardiopulmonary compromise was diagnosed if patient had (Table 1): low cardiac index; defined as cardiac index $<2.4$ $1 / \mathrm{min} / \mathrm{m}^{2}$ with low SVI $\left(<40 \mathrm{~mL} /\right.$ beat $\left./ \mathrm{m}^{2}\right)$, high pulmonary capillary wedge pressure (PCWP), defined as PCWP $>18$ $\mathrm{mm} \mathrm{Hg}$; elevated RAP, defined as RAP > $12 \mathrm{mmHg}$; elevated systemic vascular resistance index (SVRI), defined as SVRI $>2400$ dynes.sec. $\mathrm{cm}^{5} / \mathrm{m}^{2}$. Patients without these findings were characterized as non-cardiac.

\section{Statistical Analysis}

Descriptive results were characterized as either mean \pm standard deviation for normally distributed continuous variables and frequency (percent) for discrete data. Cross tabulations were used to compare determinations on the basis of TTE and RHC versus GS assessments. These provided estimates of PPV and NPV.

In situations where the two-way tables comparing agreement were symmetric, Cohen's kappa statistics for agreement [18] were calculated in order to measure overall agreement between TTE and GS, and between RHC and GS assessments. The Cramer's contingency coefficient [19] was used to estimate agreement in non-symmetric tables where the number or type of rows did not match the columns. All comparisons used a level of significance (alpha) of 0.05 . Analysis was carried out using SPSS 13.0 (SPSS Inc, Chicago).

\section{RESULTS}

\section{Patients}

All patients admitted to MICU and SICU with cardiopulmonary illness during study period were screened for enrollment. Patients who met study inclusion were enrolled in the study. RHC and TTE studies were performed in 56 consecutive patients who met the inclusion criteria. In five excluded patients the data was considered uninterpretable (three due to poor quality of RHC recordings and two due to poor TTE imaging quality). Analysis was performed on the remaining 51 patients. Table 2 shows the demographic and clinical characteristics. 35 patients $(68 \%)$ required mechanical ventilation. Severe sepsis was the most common initial diagnosis. 31 patients $(61 \%)$ were alive on discharge from ICU. Overall, 15 patients had cardiovascular compromise only, 25 had pulmonary compromise only, and 11 had both. 14 patients $(27 \%)$ had cardiac etiology of cardiopulmonary compromise as per gold standard assessment.

\section{Agreement in Etiology of Cardiopulmonary Compromise}

There was complete agreement between TTE data and RHC data in determining the etiology of cardiopulmonary compromise as cardiac or non-cardiac in $46(90 \%)$ of the 51 patients.

The kappa statistic for the agreement between TTE and GS in determining the etiology of cardiopulmonary compromise as cardiac or non-cardiac was $0.90 \quad(95 \%$ confidence interval, 0.73 to $0.98 ; \mathrm{p}<0.001)$. The kappa statistic for the agreement between RHC and GS was 0.84 (95\% confidence interval, 0.63 to $0.95 ; \mathrm{p}<0.001$ ). Based on the results of the gold standard assessment, the positive predictive value (PPV) and negative predictive value (NPV) for TTE determining the etiology of cardiopulmonary 
compromise were $93 \%$ (95\% confidence interval 0.66 to 1.0 ) and $97 \%$ (95\% confidence interval 0.86 to 1.0 ) respectively. The PPV and NPV for RHC were $100 \%$ (95\% confidence interval 0.72 to 1.0 ) and $92 \%$ (95\% confidence interval 0.80 to 0.98 ) respectively (Table 3 ).

Table 2. Patients Characteristics

\begin{tabular}{|l|l|}
\hline \multicolumn{1}{|c|}{ Characteristics } & \multicolumn{1}{c|}{ All Patients $(\mathbf{N}=\mathbf{5 1})$} \\
\hline \hline Gender Male/Female & $31 / 20$ \\
\hline Age (years) ${ }^{\mathrm{a}}$ & $67.5 \pm 8.8(27-93)$ \\
\hline Admission diagnoses & \\
\hline Severe sepsis ${ }^{\mathrm{b}}$ & $22(43 \%)^{\mathrm{b}}$ \\
\hline Pneumonia & $14(27 \%)$ \\
\hline Post-operative & $9(18 \%)$ \\
\hline Pancreatitis & $2(4 \%)$ \\
\hline Others & $4(8 \%)$ \\
\hline Mechanical Ventilation & $35(68 \%)$ \\
\hline Vasopressor use & $26(51 \%)$ \\
\hline Inotropic use & $9(18 \%)$ \\
\hline Average ICU LOS (days) ${ }^{\mathrm{a}}$ & $12 \pm 6.5($ range 3-32) \\
\hline ICU Survival & $31(61 \%)$ \\
\hline
\end{tabular}

ICU: intensive care unit; LOS: length of stay.

${ }^{\mathrm{a}}$ Values are given as mean $\pm \mathrm{SD} ;{ }^{\mathrm{b}}$ frequency (\%).

The kappa statistic for the agreement on etiology of cardiopulmonary compromise between initial clinical assessment and gold standard assessment was 0.27 (95\% confidence interval, 0.13 to $0.47 ; \mathrm{p}<0.053$ ).

\section{Diagnostic Disparities between TTE and RHC}

Three patients had a final diagnosis of major pulmonary embolism (two by chest CT angiogram and one by ventilation / perfusion scan). In two patients, RHC data was not suggestive of PE while TTE was highly suggestive. TTE showed severe RV dilatation compressing the left ventricle in diastole with pulmonary hypertension and normal LV function. (One patient had previous TTE a week before entry into the study that showed normal RV function.) In both instances, RHC data was not helpful, showing slightly decreased CI (2.2 and $\left.2.3 \mathrm{l} / \mathrm{min} / \mathrm{m}^{2}\right)$ with normal PCWP (13 and $15 \mathrm{~mm} \mathrm{Hg}$ ). One patient in shock had a diagnosis of cardiac tamponade made by TTE. Although this patient had severe sepsis from underlying infection, RHC data did not indicate cardiac tamponade. TTE findings resulted in pericadiocentesis and drainage, an important change in management for this patient.

Overall, in five cases there was disparity between RHC and TTE in establishing the etiology of cardiopulmonary compromise (Table 3). In four cases, the etiology was determined to be non-cardiac by RHC, and cardiac by TTE and in one case the etiology was determined to be cardiac by RHC and non-cardiac by TTE. Gold Standard assessment reviewed by an intensivist not caring for the patients concluded that TTE was more accurate than RHC in determining etiology of cardiopulmonary compromise in four cases (two with PE, one with cardiac tamponade, and one with renal failure and fluid overload), while RHC was more accurate than TTE in one case (patient with renal failure and fluid overload).

\section{Agreement on Initial Treatment Strategy}

In patients with cardiopulmonary compromise, overall agreement on initial treatment strategy between TTE and RHC was 46/51 (90\%).

TTE agreed with GS on initial treatment strategy in 48 of 51 cases $(94 \%)$. The contingency coefficient for agreement was 0.93 (95\% confidence interval, 0.81 to $0.99, \mathrm{p}<0.001$ ). RHC agreed with GS on initial treatment strategy in 47 of 51 cases $(92 \%)$. The contingency coefficient for agreement was $0.91(\mathrm{CI}=0.81-0.99, \mathrm{p}<0.001)$.

\section{Complications}

There were no complications during TTE procedures. Duration of RHC ranged between 24-96 hours. Complications occurred in $15 \%$ of RHC procedures. The most common complication was cardiac arrhythmias. 6 patients had non-sustained ventricular tachycardia during RHC insertion. One patient had sustained ventricular tachycardia one day after RHC insertion which resolved after removal of the catheter. One patient had transient right bundle branch block during PA catheter insertion and required no treatment. There was no pneumothorax related to vascular access insertion. Catheter related infection was not monitored during the study.

\section{DISCUSSION}

Our study confirms that a snapshot bedside TTE, when done promptly, provides substantial information about the

Table 3. Overall Agreement in Etiology of Cardiopulmonary Compromise Between Transthoracic Echocardiography and Right Heart Catheterization vs Gold Standard Assessment in 51 Patients

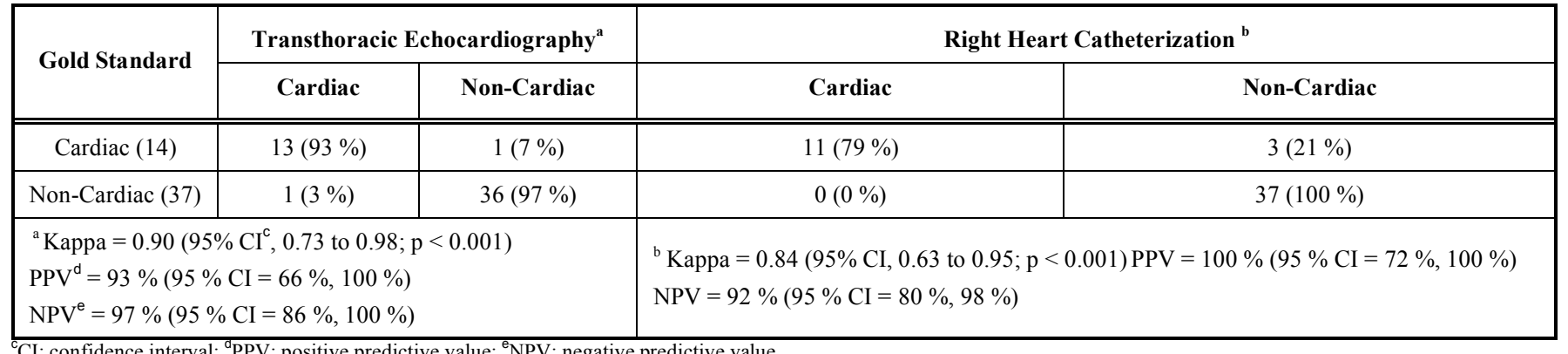

CI: confidence interval; ${ }^{\circ} \mathrm{PPV}$ : positive predictive value; ${ }^{\circ} \mathrm{NPV}$ : negative predictive value. 
etiology of severe cardiopulmonary compromise. TTE correctly classified the origin of cardiopulmonary compromise in $90 \%$ of cases compared to clinical gold standard assessment. Also, there was complete agreement between TTE data and RHC data in determining the etiology of cardiopulmonary compromise as cardiac or non-cardiac in $90 \%$ of the cases. Moreover, in $94 \%$ of cases there was agreement on initial treatment strategy between TTE and gold standard. Initial clinical assessment incorrectly identified etiology of cardiopulmonary compromise in $73 \%$ of the cases. Our results are consistent with previous reports that showed poor correlation between initial clinical assessment and right heart catheterization ranging between $30-50 \%[20,21]$. We enrolled patients who had unclear etiology of severe cardiopulmonary compromise after a trial of empiric therapy in the ICU. Our objective was not to determine hemodynamics such as cardiac output obtained by TTE and compare them to hemodynamics obtained by RHC but rather to investigate whether assessment of cardiac size and function non-invasively by TTE could provide useful and necessary information in the initial evaluation of critically ill patients with cardiopulmonary compromise. Our study design likely represents current practice in most ICUs where PA catheters are less utilized, and reserved for patients with unclear etiologies after a trial of unsuccessful empiric therapy.

We found TTE to be of diagnostic quality in $91 \%$ including patients on mechanical ventilation. Our findings are in agreement with recently published studies by Joseph and Bossone $[7,22]$ who found TTE to be technically adequate in $99 \%$ of cases, better than previous studies [23, $24,25]$ that reported only $36-50 \%$ of TTE studies as being technically adequate. This discrepancy in results is likely due to improvement in technology, use of harmonic imaging, and performance by experienced TTE technicians including after-hours studies.

We found a cardiac etiology of cardiopulmonary compromise in $27 \%$ of patients in our medical / surgical ICU. Our results are consistent with previously reported results that showed cardiac abnormalities in $36-59 \%$ of patients [7, 8]. Bossone, et al. [22] found one or more cardiac abnormalities in a significant proportion of patients (36\%) admitted to MICU when assessed by TTE, and cardiac abnormalities were unsuspected in $77 \%$ of these patients. Kaul, et al. [8] found cardiac etiology of shock or pulmonary edema in $59 \%$ of patients. Cardiac abnormalities and a predominantly cardiac etiology for cardiopulmonary compromise are thus common in the non-cardiac admissions to medical and surgical ICUs increasing underscoring the usefulness of TTE. TTE may have unique advantages over RHC in some circumstances such as suspected major PE or pericardial effusion. In our study, three patients had such diagnoses exclusively made by TTE.

Few studies have assessed the therapeutic impact of echocardiographic (TTE or TEE) on patients' management and found it to be relevant $[7,23,24]$. Our study showed excellent agreement between TTE-guided initial treatment strategy and the gold standard. This was comparable to agreement to initial treatment strategy guided by RHC.

Our study has some limitations. First, we did not have inter-observer TTE evaluation, although TTE studies were read and data sheets were filled by pre-specified criteria. Second, our study results may not be generalizable when less experienced echocardiographer or small hand held transthoracic ultrasound devices are used, although, experience with TTE image acquisition and interpretation are improving among intensivists. A recent study by Melamed et al. [26] found 0.72 kappa statistics for the agreement between a minimally trained intensivist and an echocardiographer for any abnormality in LV function. This study was limited to the evaluation of LV systolic function and did not assess agreement between intensivists and echocardiographers in patients with shock and pulmonary edema due to causes other than LV systolic dysfunction. Also, it is worth noting that our study incorporated Doppler echocardiographic data acquired for estimation of left atrial pressure, pulmonary artery pressure, and mitral regurgitation, which might have contributed to the excellent diagnostic and therapeutic agreement. Doppler echocardiography data provides additional diagnostic information beyond that provided by two-dimensional imaging of anatomy and function in the critically ill. Third, our study did not utilize Transesophageal Echocardiography (TEE), which was found to have high agreement in few previously reported studies [24, 25, 27]; however, we have found that TTE was of acceptable quality in most cases $51 / 56$ (91\%). Because of its semi-invasive nature TEE examination was found to be contraindicated in up to $15 \%$ of critically ill patients in some series [7]. Fourth, in our study, gold standard was defined by ICU physicians by coupling clinical data, echocardiographic data, and RHC data. The final diagnosis might have been biased by the belief or personal preference of the intensivist caring for the patient. On the other hand, we believe the intensivist who is caring for the patient on a daily basis and has access to all clinical, TTE, and RHC data has the most objective judgment to render. We also provided additional review by an independent intensivist of the cases when there was disparity between RHC and TTE in establishing the etiology of cardiopulmonary compromise. Fifth, although we found excellent agreement on intervention between TTE and gold standard, the therapeutic interventions recommended based on TTE data were not tested in terms of efficacy and tolerance. Sixth, our study addressed "snap shot" assessment of cardiac function in the critically ill patients and did not include continuous monitoring of cardiac function which may be desirable in some clinical circumstances. Miniaturized esophageal probes may facilitate monitoring cardiac function over a prolonged time period but commercially available miniaturized probes allow Doppler assessment of cardiac function only [28, 29].

\section{CONCLUSION}

In this era where RHC is less frequently used and intensivists are increasingly utilizing TTE to manage critically ill patients with cardiopulmonary compromise, we found that TTE is a safe and useful diagnostic tool in determining the etiology of cardiopulmonary compromise and providing treatment recommendations in critically ill patients when initial clinical assessment is limited. Our data does not exclude the use of RHC in clinical situations where there is still ambiguity after 2-D echocardiography. However, in the largest number of ICU cases 2-D 
echocardiography with Doppler will be applied first, and our data reports a real-world assessment of how often this snapshot echo will provide the correct diagnosis of severe cardiopulmonary compromise. While both methods can be complementary to each other, bedside transthoracic echocardiography is an acceptable non-invasive alternative to right heart catheterization in determining the etiology of cardiopulmonary compromise in most critically ill patients when initial clinical assessment is limited. Our results provide additional support for the increase use of TTE and the decreasing use of RHC in the management of critically ill patients with cardiopulmonary compromise reported in recent years. Intensivists may utilize goal directed echocardiographic data for diagnosis and management of the critically ill.

\section{AUTHORS' CONTRIBUTIONS}

HK has made significant contributions to study conception and design, acquisition of data, analysis and interpretation of data, and has been involved in drafting and revising of the manuscript. EE has made significant contributions to study design, analysis and interpretation of data, and has been involved in drafting and revising of the manuscript. PH has made significant contributions to study design, analysis and interpretation of data, and has been involved in drafting and revising of the manuscript. MS has made significant contributions to study conception and design, analysis and interpretation of data, and has been involved in drafting and revising of the manuscript. All authors read and approved the final manuscript.

\section{ACKNOWLEDGEMENT}

Declared none.

\section{CONFLICT OF INTEREST}

Declared none.

\section{ABBREVIATIONS}

$\begin{array}{ll}\mathrm{CI} & =\text { Confidence interval } \\ \mathrm{CA} & =\text { Clinical assessment } \\ \mathrm{GS} & =\text { Gold Standard } \\ \mathrm{ICU} & =\text { Intensive care unit } \\ \mathrm{IVC} & =\text { Inferior vena cava } \\ \text { LV } & =\text { Left ventricle } \\ \text { LVEDA } & =\text { Left ventricular end diastolic area } \\ \text { LVESA } & =\text { Left ventricular end-systolic area } \\ \text { NPV } & =\text { Negative predictive value } \\ \text { PAC } & =\text { Pulmonary artery catheter } \\ \text { PCWP } & =\text { Pulmonary capillary wedge pressure } \\ \text { PE } & =\text { Pulmonary embolism } \\ \text { PPV } & =\text { Positive predictive value } \\ \text { PVF } & =\text { Pulmonary vein flow } \\ \text { RHC } & =\text { Right heart catheterization } \\ \text { SVI } & =\text { Stroke volume index } \\ \text { TEE } & =\text { Transesophageal echocardiography } \\ \text { TTE } & =\text { Transthoracic echocardiography }\end{array}$

\section{REFERENCES}

[1] Connors AF, Dawson NV, McCaffree R, et al. Assessing hemodynamic status in critically ill patients: do physicians use clinical information optimally? J Crit Care 1987; 2: 174-80.

[2] Sandham JD, Hull RD, Brant RF, et al. A randomized, controlled trial of the use of pulmonary-artery catheters in high-risk surgical patients. N Engl J Med 2003; 348:5-14

[3] Harvey S, Harrison DA, Singer M, et al. Assessment of the clinical effectiveness of pulmonary artery catheters in management of patients in intensive care (PAC-Man): a randomized controlled trial. Lancet 2005; 366: 472-77.

[4] C Richard C, Warszawski J, Anguel N, et al. Early use of the pulmonary artery catheter and outcomes in patients with shock and acute respiratory distress syndrome. JAMA 2003; 290: 2713-20.

[5] Connors AF Jr, Speroff T, Dawson NV, et al. The effectiveness of right heart catheterization in the initial care of critically ill patients. JAMA 1996; 276: 889-97.

[6] Dalen JE, Bone RC. Is it time to pull the pulmonary artery catheter? JAMA 1996; 276: 916-18.

[7] Joseph MX, Disney PJ, Costa RD, et al. Transthoracic echocardiography to verify or exclude cardiac cause of shock. Chest 2004; 126: 1592-7.

[8] Kaul S, Stratienko AA, Pollock SG, et al. Value of twodimensional echocardiography for determining the basis of hemodynamic compromise in critically ill patients. J Am Soc Echocardiogr 1994; 7: 598-606.

[9] Stamm RB, Carabello BA, Mayers DL, et al. Two-dimensional echocardiographic measurement of left ventricular ejection fraction: Prospective analysis of what constitutes an adequate determination. Am Heart J 1982; 104: 136-44.

[10] Brennan JM, Blair JE, Goonewardena S, et al. Reappraisal of the use of the inferior vena cava for estimating right atrial pressure. $\mathrm{J}$ Am Soc Echocardiogr 2007; 20: 857-61.

[11] Bendjelid K, Romand JA, Walder B, et al. Correlation between measured inferior vena cava diameter and right atrial pressure depends on echocardiographic method used in patients who are mechanically ventilated. J Am Soc Echocardiogr 2002; 15: 944-9.

[12] Feissel M, Michard F, Faller JP, et al. The respiratory variation in inferior vena cava diameter as a guide to fluid therapy. Intensive Care Med 2004; 30: 1834-7.

[13] Barbier C, Loubières Y, Schmit C, et al. Respiratory changes in inferior vena cava diameter are helpful in predicting fluid responsiveness in ventilated septic patients. Intensive Care Med 2004; 30: 1740-6.

[14] Nishimura RA, Tajik AJ. Evaluation of diastolic filling of left ventricle in health and disease: Doppler echocardiography is the clinician's Rosetta Stone. J Am Coll Cardiol 1997; 30: 8-18.

[15] Yamamoto K, Nishimura RA, Chaliki HP, et al. Determination of left ventricular filling pressure by Doppler echocardiographer in patients with coronary artery disease: Critical role of left ventricular systolic function. J Am Coll Cardiol 1997; 30: 1819-26. Come PC, Duchsook K, Parker AJ, et al. Early reversal of right ventricular dysfunction in patients with acute pulmonary embolism after treatment with intravenous tissue plasminogen activator. J Am Coll Cardiol 1987; 10: 971-8.

Jardin F, Dubourg O, Gueret P, et al. Quantitative two-dimensional echocardiography in massive pulmonary embolism: emphasis on ventricular interdependence and leftward septal displacement. J Am Coll Cardiol 1987; 10: 1201-6.

[18] Cohen J. A coefficient of agreement for nominal scales. Educ Psychol Meas 1960; 20: 37-46.

[19] Goodman L, Kruskal W. Measures of association for crossclassifications. J Am Stat Assoc 1979; v49: 732-64.

[20] Connors AF, McCaffree DR, Gray BA. Evaluation of right-heart catheterization in the critically ill patient without acute myocardial infarction. N Engl J Med 1983; 308: 263-7.

[21] Eisenberg PR, Jaffe AS, Schuster DP. Clinical evaluation compared to pulmonary artery catheterization in hemodynamic assessment of critically ill patients. Crit Care Med 1984; 12: 54953.

[22] Bossone E, DiGiovine B, Watts S, et al. Range and prevalence of cardiac abnormalities in patients hospitalized in a medical ICU. Chest 2002; 122: 1370-6.

[23] Heidenreich PA, Stainback RF, Redberg RF, et al. Transesophageal echocardiography predicts mortality in critically patients with unexplained hypotension. J Am Coll Cardiol 1995; 26: 152-8. 
[24] Vignon P, Mentec H, Terre S, et al. Diagnosis accuracy and therapeutic impact of transthoracic and transesophageal echocardiography in mechanically ventilated patients in the ICU. Chest 1994; 106: 1829-34.

[25] Hwang JJ, Shyu KG, Chen JJ, et al. Usefulness of transesophageal echocardiography in the treatment of critically ill patients. Chest 1993; 104: 861-6.

[26] Melamed R, Sprenkle MD, Ulstad VK, et al. Assessment of left ventricular function by intensivists using hand-held echocardiography. Chest 2009; 135: 1416-20.
[27] Benjamin E, Griffin K, Leibowitz AB, et al. Goal-directed transesophageal echocardiography performed by intensivists to assess left ventricular function: comparison with pulmonary artery catheterization. J Cardiothorac Vasc Anesth 1998; 12: 10-5.

[28] Dark PM, Singer M. The validity of trans-esophageal Doppler ultrasonography as a measure of cardiac output in critically ill adults. Intensive Care Med 2004; 30: 2060-6.

[29] De Vaumas C, Lafanechere A, Kermarrec N, et al. Assessment of preload and contractility using continuous esophageal Doppler cardiac output monitoring as a tool to optimize vasoactive support in septic shock. Crit Care Med 2001; 95: A427.

(c) Khouli et al.; Licensee Bentham Open.

This is an open access article licensed under the terms of the Creative Commons Attribution Non-Commercial License (http://creativecommons.org/licenses/ by-nc/3.0/) which permits unrestricted, non-commercial use, distribution and reproduction in any medium, provided the work is properly cited. 